\section{$\underset{\substack{\text { hommes } \\ \text { \& migrations }}}{ }$}

\section{Hommes \& migrations}

Revue française de référence sur les dynamiques

migratoires

$1311 \mid 2015$

Femmes et migrations

\title{
« Des mots pour la MEEAO » : poétiser et politiser la ville
}

\section{Marion Dupuis}

\section{(2) OpenEdition \\ Journals}

\section{Édition électronique}

URL : http://journals.openedition.org/hommesmigrations/3355

DOI : 10.4000/hommesmigrations.3355

ISSN : 2262-3353

\section{Éditeur}

Musée national de l'histoire de l'immigration

\section{Édition imprimée}

Date de publication : 1 juillet 2015

Pagination : 156-159

ISBN : 978-2-919040-32-2

ISSN : $1142-852 X$

\section{Référence électronique}

Marion Dupuis, « «Des mots pour la MEEAO » : poétiser et politiser la ville», Hommes \& migrations [En ligne], 1311 | 2015, mis en ligne le 09 février 2016, consulté le 14 septembre 2020. URL : http:// journals.openedition.org/hommesmigrations/3355 


\title{
MÉMOIRES
}

\section{"DES MOTS POUR LA MEEAO" POÉTISER ET POLITISER LA VILLE}

\author{
par MARION DUPUIS, ingénieure d'études, EHESS.
}

“... Mais qui tourne ma voix, qui écorche ma voix? Me fourrant dans la gorge mille crocs de bambou. Mille pieux d'oursin. C'est toi sale bout du monde. Sale bout de petit matin; C'est toi sale haine.

C'est toi poids de l'insulte et cent ans de coups de fouet.

C'est toi cent ans de ma patience cent ans de mes soins juste à ne pas mourir.

Rooh oh..."

Aimé Césaire - Extrait de Cahier d'un retour au pays natal. Poème inscrit au pochoir sur l'une des fenêtres emmurées de l'immeuble de la MEEAO.

$E^{n}$ n novembre 2010, le collectif artistique Paris Label' décida d'investir symboliquement les façades emmurées de la MEEAO. II ne restait alors plus que ces vestiges muets portant témoignage de la seconde expulsion des habitants. Ensemble, les membres du collectif - Paule Kingleur, Rémy Bovis, Anne Maurange, Cyrille Bosc, Capucine Fouga, Esther Yaya, Jérôme Barbosa, Claire Sousbie - ont créé une œuvre poétique, œuvre d'art in situ qui s'inscrit dans la mouvance du street art, où l'on s'adresse directement aux passants sans intermédiaire institutionnel.
L'intervention poétique Des mots à la MEEAO puise son inspiration dans l'histoire - passé colonial et mouvement de la négritude - et dans les mémoires croisées qui s'enchevêtrent. Elle porte une réflexion sur l'histoire locale, sur le pouvoir des mots, l'engagement politique, ainsi que sur les rapports entre espace et temps.

Le quartier de la Porte Dorée, où se situe l'immeuble de la MEEAO, conserve à ce jour de nombreuses traces de l'époque coloniale, qu'il s'agisse de l'ex-Palais des colonies devenu Musée national de l'histoire de l'immigration ou des anciens pavillons de l'Exposition coloniale adossés au lac Daumesnil. Les noms de plusieurs rues du quartier témoignent aussi de cet héritage historique². Comme l'écrit Francis Sessou, architecte et passeur de mémoire de la MEEAO : "Le lien est à ce point organique que la MEEAO ne peut se penser en dehors du contexte urbain et socio-culturel originel qui l'a vu naitre. Témoin d'un passé aux contours disparus, la résidence de la Porte Dorée serait peutêtre bien, aux côtés du Palais de la Porte Dorée (l'ex-Musée des colonies donc), l'ultime vestige d'une époque révolue, le troisième sommet d'une triangulation reliant de manière organique: le Bois, où furent exhibées les colonies, le Musée des colonies et, enfin, la résidence de certains représentants des colonies 3 ." 
Installation sur les murs et fenêtres murées de la MEEAO après l'évacuation

des résidents, installation Paris Label - Paule Kingleur. (C) Sylvie Boitel, novembre 2010.

\section{L’art du témoignage}

Des mots à la MEEAO est le fruit d'une intervention artistique nocturne qui a vu le jour en novembre 2010. Au cœur de l'œuvre, l'idée de reproduire des poèmes d'Aimé Césaire et de Léopold Sédar Senghor transcrits au pochoir, présentant des volées de mots blancs contrastant avec la grisaille des briques. Les poèmes qui surgissaient de fenêtres emmurées étaient reliés par des photographies et des listes de noms d'anciens habitants formant des frises. Des silhouettes semblaient surgir de l'opacité des murs pour interpeller, apostropher et exhorter les passants. Paule Kingleur, à l'initiative de cette action collective, explique que certains poèmes furent choisis pour leur force, leur grande violence, alors que d'autres textes sont plus consensuels et accessibles tels "Poème à mon frère blanc" de Senghor. On ajouterait que certains poèmes portent une dimension stellaire, presque surréaliste.

C'est par ce texte - à la fois court et fort - que les artistes expliquent leur démarche : "Des mots à la MEEAO... La peau des murs transpire et témoigne. En hommage aux résidents illustres et anonymes qui séjournèrent dans cette maison. Cette maison qui vit passer les poètes Léopold Sédar Senghor, Aimé Césaire, les intellectuels Cheikh Anta Diop, Alioune Diop... mais aussi des hommes politiques et des musiciens, des étudiants, des familles et des enfants des États d'Afrique de l'Ouest... Cette maison qui connut les grandes heures et débats des indépendances et de la négritude... Cette maison à présent murée, murmures emmurés... Cette maison dont nous partageons l'histoire, la mémoire collective... Cette maison qui est notre patrimoine à tous... Cette maison qui appelle le respect, la vigilance, l'engagement civique... Nous, artistes, simples citoyens, nous avons choisi le témoignage poétique comme 


\section{MÉMOIRES}

expression d'une mémoire qui ne peut être murée, escamotée, ignorée, méprisée, pour une maison dont l'avenir nous concerne... Et puis pour ne pas oublier que certains de nos voisins de la MEEAO, enfants et petits-enfants des 'combattants-tirailleurs sénégalais', se retrouvent en errance, loin de leur travail, déplacés, dans l'anxiété des décisions administratives ${ }^{4} . . . "$

L'art permet de revisiter et de transmettre la mémoire de ce lieu emblématique qu'est la MEEAO, dans sa dimension historique symbolique et stratégique.

\section{Des murs chargés d'histoire}

Paule Kingleur explique : "J'ai appelé ça Des mots à la MEEAO, car il me semblait que ces murs devaient transpirer de l'histoire des gens qui y étaient passés. Il y a eu les grands débats à la MEEAO sur la négritude et sur l'indépendance, c'est quelque chose de très important, c'est une richesse incroyable. (...) Ces textes ont aussi un sens par rapport au départ de tous les habitants, des familles, des enfants. Le contexte qui a vu naitre ce projet, c'est d'abord la force des expulsions des sans-papiers. Et le contraste entre, d'un côté, une période où Nicolas Sarkozy rendait hommage à Aimé Césaire au Panthéon, et, de l'autre côté, l'on ne s'occupait pas de l'immeuble de la MEEAO qui représente pourtant un réel patrimoines."

L'expulsion des habitants et le combat pour perpétuer la mémoire de la maison s'inscrivent aussi dans un autre contexte historique, celui de l'anniversaire des indépendances. C'est ce qu'explique un ancien habitant de l'immeuble, Ousmane Ba : "Ça fait mal quand même de perdre une maison comme ça, une maison qui est chargée d'histoire, ce n'est pas une maison qu'on a squattée, c'est une maison qui a été achetée par les États africains à la sueur des paysans sénégalais, avec le café de Côte d'Ivoire, le coton du Burkina Faso6."

Plus largement, Des mots à la MEEAO porte une réflexion implicite sur l'histoire. Le mouvement de la négritude affirmait qu'il fallait être soimême et être porteur de sa propre histoire? ${ }^{7}$ luttant ainsi contre les représentations selon lesquelles les pays colonisés ont échappé à l'histoire.

\section{Le jeu des symboles}

La démarche artistique s'attache à des symboles que l'on tente de revisiter. En premier lieu, c'est la force et l'évidence du mur de briques obstruant les fenêtres qui inspirèrent l'élan créatif. Paule Kingleur l'évoque en ces termes: "lly a eu, tout d'abord, la violence de l'expulsion des habitants, dont nous avons été témoins et qui nous a marqués et, ensuite, ce qui en est resté, des murs... Pour moi, le mur est une image forte. Pour conserver la mémoire de ce lieu et de tous les habitants qui s'y sont succédé, j'ai pensé qu'il serait intéressant d'en faire des fenêtres murées mais ouvertes sur le monde, des fenêtres d'écriture, des fenêtres de poèmes, qui seraient sans doute régulièrement recouvertes, mais sur lesquelles on pourrait écrire de nouveau. On n'aurait de cesse de faire parler ces murs, de leur donner la parole."

L'autre espace éminemment symbolique de l'immeuble se situe au sous-sol et revêt la forme d'une grande salle collective. Ce lieu abrita les grands débats politiques des années 1950 et 1960, mais aussi toutes sortes d'événements du quotidien, fêtes, mariages, bals et cours de sports. Cet espace est en quelque sorte l'âme du lieu. Les plans de reconstruction de l'immeuble ne prévoient pas que I'on conserve cet espace collectif du vivre- 
ensemble, ce que regrette Paule Kingleur. Elle ne revendique pas non plus la notion de lieu de mémoire pour la MEEAO. Ce terme lui semble figé dans le passé, la mémoire doit être vivante et non culpabilisante. Elle aurait souhaité qu'un espace collectif puisse subsister, ce qu'elle nomme "lieu de palabres", un lieu où l'on échange et où l'on partage et qui fait le lien entre différentes histoires.

L'idée qui sous-tend cette action artistique est aussi de créer un espace d'expression alternatif où l'on célèbre le mouvement de la négritude et la présence africaine en France, mais aussi où se confondent tous les résidents de l'immeuble, qu'ils soient illustres ou exclus de la République, pour clairement donner une portée sociale et politique au projet. Ainsi, que l'on mêle dans un même ensemble des personnes qui occupèrent des postes à responsabilités au sein de l'État français à d'autres rendus totalement invisibles par leur statut de sans-papiers. Paule Kingleur relate cette anecdote qui relie personnages illustres et anonymes et dont le seul lien n'est autre que la MEEAO. "Nous avions inscrit des poèmes sur les deux façades, côté boulevard Poniatowski et côté rue Claude-Decaen. Tous les poèmes du boulevard Poniatowski ont été repeints, camouflés d'une couleur beige, pour ne pas dire censurés. Quand je suis repassée devant, j'ai vu qu'un nom d'auteur était resté tout en bas du mur, on pouvait distinguer la signature 'Léopold Sédar Senghor'. Ça m'a amusée car je me suis dit que la personne chargée de repeindre, qui le faisait sous ordre, avait peut-être décidé à son tour de laisser une trace."

\section{Un espace stratégique}

La maison n'est réellement connue que des gens du quartier, ancrée dans son espace local. Paule
Kingleur parle de la MEEAO comme d'un village dont elle connaissait les moindres détours, les moments quotidiens et la grande histoire. Son intérêt pour ce lieu est aussi lié à son attirance pour l'Afrique - elle y a vécu et travaillé par épisodes durant vingt ans - et aussi pour son amour des grands poètes, notamment Césaire. Et puis il y a l'envie de transmettre, de faire connaître aux gens du quartier. "Je trouve que les mots, c'est ce qu'on lit le plus facilement, mais je ne voulais pas de textes militants, portant des revendications sociales. Je voulais passer par des poètes pour pouvoir toucher les gens. C'est émouvant de les voir s'arrêter, lire la colère poétique d'Aimé Césaire ou les textes plus ronds de Léopold Sédar Senghor. Ce témoignage poétique sur la façade de la MEEAO représente une forme de militantisme qui me correspond : un engagement dans la rue avec ma propre expression. Les textes choisis reflètent la violence qu'il peut y avoir dans la société dominante, la violence qu'il y a dans l'acte même de coloniser. Mais cette violence peut aussi être sournoise, discrète. Ce projet artistique est un médium pour parler aux habitants du quartier. II y a un illot d'électeurs du Front national dans un immeuble proche de la MEEAO. Une sorte de lutte d'influence s'est instaurée dans l'espace. Écrire sur les murs, pour nous, c'est une manière de mener le combat."

Le combat des artistes de Paris Label pour la transmission de la mémoire de la MEEAO a porté ses fruits. Le cabinet d'architectes chargé de la rénovation de l'immeuble a prévu d'orner les murs des couloirs de poèmes transcrits par une multitude de lettres de petites tailles. Reprenant des poèmes choisis par Paris Label et d'autres plus récents, s'inspirant de l'idée tout en proposant une nouvelle graphie, ce projet architectural propose une forme renouvelée d'écriture sur les murs de la MEEAO. 